\title{
Performance Analysis of Cognitive Radio Based Internet-Of-Things Network for Energy Efficiency and Spectrum Utilization
}

\author{
Palak Thakur, Payal Patial, Prabhat Thakur
}

\begin{abstract}
Energy and Spectrum are the two basic requirements in the realm of Internet-of-Things (IoT). The network of IoT is becoming larger day by day and the design of spectrum and energy efficient solution is a quite challenging task because of the rapid increase of connecting devices in IoT network. To make the system more energy and spectral efficient, energy harvesting and cognitive radio (CR) are the proficient solutions, respectively. This paper introduces a spectral and energy efficient design for CR based sensor networks. We present a network architecture, in which nodes or other sensing devices can use the spectrum opportunistically and energy harvesting can be done from different ambient sources. We then propose an 1) energy alancing scheme for heterogeneous network in which nodes will have different energy levels and 2) Cluster head (CH) selection scheme which will only be performed on the few nodes of network having the highest current energy to accomplish the ultimate goal of energy balancing in network, this analysis is performed with in the cluster. Furthermore, for the spectral efficiency, we propose a channel management scheme based on cognitive radio to allot the best available channel having highest reliability in respect of the bit error rate (BER) using. Comprehensive results exhibit the effectiveness in the performance of the proposed spectral and energy efficient schemes and show better performance over other schemes.
\end{abstract}

Index Choice: Channel management, clustering, cognitive Radio, energy management, Internet-of-Things,

\section{INTRODUCTION}

$\mathrm{T}$ The Internet-of-things (IoT) is a potential technology leading towards the new era of communication which facilitate connectivity among physical objects [1]. Devices in this network are increasing day by day thus the IoT networks are facing the challenges like need of spectrum and energy requirement. The primary cause for these sort of difficulties isa huge number of devices in the network. Cognitive Radio and Energy Management Techniques are the two efficient methods to overcome the problem of spectrum shortage and energy balancing respectively.

${ }_{1}$ Revised Manuscript Received on July 02, 2019.

Palak Thakur, M.E student, Department of Electronics\& Communication Engineering, Chandigarh University, Gharuan.

Payal Patial, Professor, Departmentof Electronics \& Communication Engineering, Chandigarh University, Gharuan.

Dr. Prabhat Thakur, Post Doctoral Research Fellow, Department of Electrical and Electronics Engineering, University of Johannesburg, S.A
- Cognitive Radio is a efficient solution to overcome the problem of spectrum sharing [2]-[4] by allowing the unlicensed users to coexist with licensed users [1], [5]. Cognitive Radio use available spectrum which makes it spectrum efficient technology [6].

- Energy Management plays a crucial role in increasing the lifetime of the network by using different protocols or by using the mechanism of energy harvesting. Some of of these commonly used protocols are LEACH (Low Energy Clustering Hierarchy), Stable Election Protocol (SEP) and Zonal-stable Election Protocol (ZEP)[7].Energy Harvesting is a another technology which can increase the energy efficiency by providing the energy from ambient resources [8].

\section{A. Contribution}

In this paper, we examine the different technologies of spectral and energy efficient CRSN for IoT. We have proposed the energy efficient scheme to ameliorate the lifetime of the network by deploying the gateway nodes and making the network heterogeneous in terms of energy. The work flow of this paper is as follows:

1. Energy Management Scheme:

- $\quad$ Full operational mode (FOM)

- Dynamic operational mode(DOM)

2. $\mathrm{CH}$ selection Scheme

3. Cache Nodes

4. Channel Management Scheme

The paper is designed as follows, the related work is presented in Section II providing the description of work done in the area of IoT. Further, Section III provides the Proposed Work and the system model is presented in Section IV. The simulation results are in Section $\mathrm{V}$ and finally Section VI concludes the paper.

\section{RELATEDWORK}

- Saleem Aslam, et.al (2018) proposed a novel energy and spectrum efficient mechanism for improving spectral and energy efficiency in cognitive radio sensor networks (CRSN) [9]. The spectrum was accessed opportunistically and energy was harvested from ambient RF sources by this proposed architecture. The best quality channel in terms

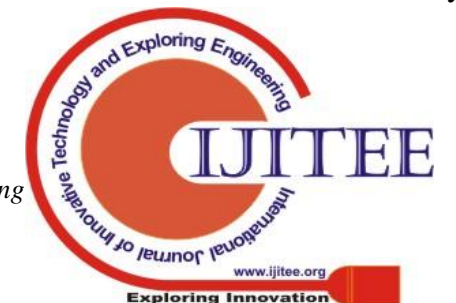


of stability and reliability was assigned to the sensor nodes by proposing a channel management mechanism to achieve reliable intra-cluster reporting. Highly effective results were achieved which showed that this approach provided highly spectrum efficient mechanism and was also better that previously existing approaches Basma

of Vertex Covers. The energy and communication costs which add up when the network is monitored are minimized through the proposed approach. As per the simulation results it was seen that appropriate energyefficient solution was achieved for monitoring IoT networks by applying proposed technique.

- $\quad$ Santiago S, et.al (2017) proposed a novel implementation and design to improve the energy efficient routing of IoT networks [11]. The performance of network was optimized by integrating the routing metrics. For selecting the preferential path and increasing the lifetime of networks, the energy aware metrics were merged by the proposed technique using fuzzy inference system. MATLAB simulator was used to perform simulations and the results achieved showed the performance level of around $63 \%$ when implementing proposed technique.

- $\quad$ S.Nisha, et.al (2017) proposed a protocol for IoT which was named as ESMR (Energy Efficient Self-organizing Multicast Routing) [12]. The nodes were considered to be the ones which already existed in the network. Multiple measurements along with Markov process were used for measuring the weight of network node in case of nonnetwork nodes.
Mostafa, et.al (2018) proposed an III-phase mathematical model for handling the optimized scheduling within IoT networks [10]. The Vertex Cover Problem was solved iteratively in the initial phase to generate the different subsets of nodes which cover the complete graph. The other two phases managed the optimized scheduling

- K. Suresh, et.al (2016) studied that for minimizing the energy Minimum Energy Consumption Algorithm(MECA), several techniques were proposed over time [13]. However, because of the direct distribution of nodes in the network, these techniques were considered to be inefficient. To control and regulate the energy factors in IoT efficiently, an Energy Efficient IoT approach was proposed named EEIoT. The energy harvesting of IoT was reduced by this self-adaptive technique. Towards the end, a comparative analysis of existing and proposed techniques on the basis of various energy consumption factors was performed which showed that the proposed approach outperformed other techniques.

- Muneer Bani Yasin, et.al (2016) proposed a dynamic tuning approach such that the update message interval within CoAP could be adjusted [14]. The battery level of the transmitting node was considered to be the base of proposed algorithm. Here, with the reduction in the battery level the update interval was maximized. Through the simulation results it was seen that around $25 \%$ of extension in the lifetime of network was achieved when implementing the proposed approach in the IoT network.

TABLE I

Summary of Literature survey

\begin{tabular}{llll}
\hline Ref. & Objective & Paper/Year & Remarks \\
\hline
\end{tabular}

[15]

Energy Management in IoT based smart homes

[16] wireless sensor network

Selection of Cluster Head using modified LEACH

$\mathrm{CH}$ selection is based on the Weights considering the average and residual energy of the nodes
RF energy harvesting based

\begin{abstract}
Transfer of power and MIMO broadcasting for information simultaneously by simultaneous wireless using MIMO technology

Amalgamated energy and spectrum management information and power transfer/2013

Integrated energy and spectrum harvesting for $5 \mathrm{G}$ wireless communications/2015
\end{abstract}

Efficient energy management for Internet-of-things in smart cities/2017

Adaptation of leach routing protocol to cognitive radio sensor networks/2012

Energy efficient clustering scheme for prolonging the lifetime of wireless sensor network with isolated nodes/2015

Energy harvesting and spectrum sharing protocol for wireless sensor networks/2015
Energy management is divided into two parts: energy harvesting and energy efficient solution

Architecture Power splitting \& Time switching are analyzed

Energy management is done by using MAC protocols

Lifetime of the network is increased by locating the $\mathrm{CH}$ in suitable location

Lifetime of the network is prolonged by selecting the energy rich node as $\mathrm{CH}$

Achieve optimal throughput and network lifetime

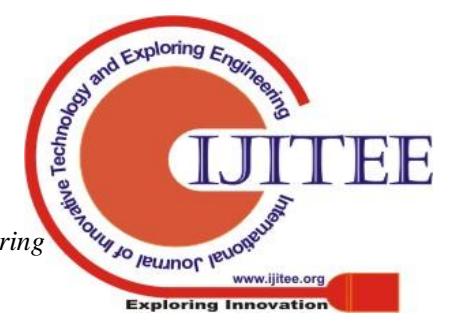


Efficient solution of energy and Energy and Spectral efficient spectrum scarcity in IoT cognitive radio sensor network for Internet-of-Things/2018
Energy efficiency has been increased and by using $\mathrm{CR}$ the opportunistic spectrum sharing is done.

Network lifetime and the residual energy is increased the cache nodes radio based Internet-of-things Network for energy efficiency and spectrum utilization

\section{NETWORK MODEL}

In this research work, the novel energy efficient approach is proposed for the better energy efficiency in IoT. The protocol which is more energy efficient is based on the approach of clustering and node heterogeneity which is explained in this section.

\section{A. Energy Efficient Model}

For an IoT framework which incorporates $\mathrm{C}$ number of clusters, $\mathrm{N}$ numbers of nodes inside each cluster, data channels are of $\mathrm{M}$ number and one control channel inside each and every cluster, a cluster based improved Cognitive Radio Sensor Network (ICRSN) is considered [22]. The sensor nodes are expected to be equipped with various wireless interfaces to such that information transmission and harvesting can be performed. The intra-cluster communication tasks are managed reliably using a different local control channel. A approach of clustering through which sensing nodes are divided into several groups utilizing k-means clustering [23]algorithm is applied for dealing with the energy and spectral variations occurring in the time and space. The transmission power is minimized through clustering which thus helps in developing the overall lifetime of networks. The transmission time includes the total time consumed in between the deployment and non-active state of ICRSN [14]. When certain number of nodes die the in network is known to be non-functional[24][25]. Following mathematical representation can be used to describe the clustering by the kmeans algorithm[23].

$$
\gamma=\sum_{l=1}^{C} \sum_{i=1}^{N} \partial_{i l}(1)
$$

Here, the distance existing betweenthe center of corresponding cluster $l$ and node $i$ is denoted by $\partial_{i l}$. The global view of ICRSNs is represented by the parameterY. The clusters are assumed to be non-overlapping. Thus, for every cluster, similar sets of channels are provided. Channel $\mathrm{k}$ can be allotted only to one node in a cluster. The feasibility that the sensor nodes of similar cluster will collide is eliminated here. Selecting the $\mathrm{CH}$ is the next prime task after cluster formation. There is a random selection of $\mathrm{CH}$ in the first step. Since the energy level of all the nodes is same, this assumption is considered as valid.

The next step applies the CH selection method [26]. Spectrum sensing, reporting and monitoring of tasks parallel with energy harvesting are the few tasks performed by each of the sensor node. Similarly, spectrum sensing, data reception and channel allocation are some of the tasks performed by $\mathrm{CH}$. Further, environment monitoring and data transmission are also performed by $\mathrm{CH}$. The figure 1 below shows the architecture of cluster-based sensor network for IoT. The spectrum band of PUs which use parameter adaptation and spectrum sensing approach of $\mathrm{CR}$ are accessed by the nodes.

The spectral utilization and efficiency are optimized by this process. The data that is gathered by the nodes is forwarded by $\mathrm{CH}$ to the gateway node which forwards it to the cloud linked to that network [27]. To store and process the received information a protocol stack is provided in the cloud. The required information can be restored from the cloud by the network manager. The sink node is assumed to lie within the transmission range of $\mathrm{CH}$. In an intra-cluster environment, energy and spectrum management are focused on such that simplicity and illustration can be handled. Similar scheme is applied to all the clusters because of the distributed nature of operations. Thus, over the entire network energy and spectral efficiency are achieved. In the HEACHS protocol, the nodes are heterogeneous in nature means the configuration of the nodes are different. The gateway nodes are used in between the base station and $\mathrm{CH}$. The $\mathrm{CH}$ will transfer the data to gateway nodes and then the gateway nodes will pass information to base station. This routing will reduce the consumption of energy in the network as compared to EMS protocol. The proposed architecture of the network for IoT is presented in Fig.1.

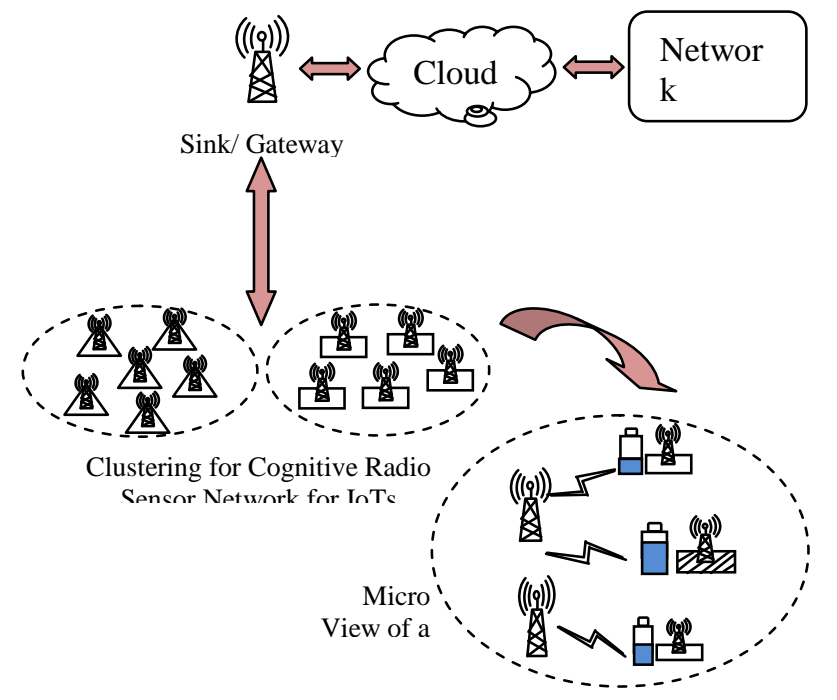

Fig.1:Energy efficient architecture for IoT

The sensor nodes and $\mathrm{CH}$ perform several tasks. The $\mathrm{CH}$ receives the Energy 
information packet EIPs that includes node ID, current levels from each node initially [28]. Thus, average energy and current levels of all the nodes will be included in $\mathrm{CH}$. For transmitting EIP, $E_{i}^{e r}$ extentt of energy is consumed by the $\mathrm{i}^{\text {th }}$ sensor node which is also called the energy reporting process. The spectrum sensing task is performed by every node after forwarding EIP. A local control channel is used by the node to report its observation. $E_{i}^{s S}$ and $E_{i}^{s r}$ are the respective energy consumption values of $i^{\text {th }}$ sensor node for the spectrum sensing and reporting. Further, the environmental monitoring task which consumes $E_{i}^{m}$ energy is executed by the sensing node. The monitoring results are transmitted from the $\mathrm{CH}$ to the $\mathrm{i}^{\text {th }}$ node once the request of reporting is received from $\mathrm{CH}$. Further, $E_{i}^{r c v}$ and $E_{i}^{d t}$ are the respective energy expenditures when reporting request is received from $\mathrm{CH}$ and for the transmission of the monitored data to $\mathrm{CH}$. The following equation (2) calculates the total amount of energy consumed by $\mathrm{i}^{\text {th }}$ node $\left(E_{i}^{\text {cons }}\right)$ during a frame as:

$E_{i}^{\text {cons }}=E_{i}^{e r}+E_{i}^{s s}+E_{i}^{s r}+E_{i}^{m}+E_{i}^{r c v}+E_{i}^{d t}+E_{i}^{i d l e}(2)$

Here, the node $i$ consumes $E_{i}^{i d l e}$ energy when it is in idle state. The sensor nodes are expected to be heterogeneous in nature in the $c_{l}$ cluster [29]. Following equation (3) calculates the energy consumed by $\mathrm{i}_{0}^{\text {th }} \mathrm{CH}\left(E_{i 0}^{\text {cons }}\right)$ in one frame from the CH's perspective as:

$$
\begin{aligned}
E_{i 0}^{c o n s}= & E_{i 0}^{r e r}+E_{i 0}^{s s}+E_{i 0}^{r s r}+E_{i 0}^{p r}+E_{i 0}^{m}+E_{i 0}^{d r q}+E_{i 0}^{d r c v}+ \\
& E_{i 0}^{i d l e / s n k r}
\end{aligned}
$$

Here, the energies used by $\mathrm{CH} \mathrm{i}_{0}$ for receiving the EIP's, gathering the spectrum sensing results from remaining sensor nodes and executing spectrum sensing tasks are represented as $E_{i 0}^{r e r}, E_{i 0}^{r s r}, E_{i 0}^{s s}$ respectively. Further, while processing the spectrum sensing reports, atmospheric monitoring, data reception and data request tasks, the amount of energy consumed by $\mathrm{CH}$ denoted by $\mathrm{i}_{0}$ is provided as $E_{i 0}^{p r}, E_{i 0}^{m}, E_{i 0}^{d r c v}, E_{i 0}^{d r q}$ respectively. The data is forwarded by the $\mathrm{CH}$ to the sink node as per the request. The data is forwarded to the sink and $E_{i 0}^{s n k r}$ energy is consumed in case if the request arrives. Otherwise, $E_{i 0}^{i d l e}$ is consumed in the idle state.

\section{B. Frame Structure}

The frames are equipped of a functional and control slots. It is interpreted that nodes are designed with a separate unit for harvesting the energy sources in environment, so that nodes can collect the energy. The $\mathrm{CH}$ is chosen for the particular numbers of frame which is stated as $\mathrm{CH}$ tenure $\mathrm{T}_{\mathrm{CH}}$. For instance, if the node $i$ is $\mathrm{CH}$ for $\mathrm{T}_{\mathrm{CH}}=20$ in frame index $\mathrm{r}=21$, at that point it can go about as $\mathrm{CH}$ from frame index $\mathrm{r}=21$ to 40. At the start of $40^{\text {th }}$ frame index, all nodes will send their average and present/ current energy levels to $\mathrm{CH}$. $\mathrm{CH}$ will select the new cluster head on the basis of defined criteria in section IV.

\section{Energy Harvesting Model}

The energy can be harvested from ambient RF sources by the sensor nodes [29]. Certain factors like the transmitting power of source, surrounding propagation properties and harvesting circuit can define the energy harvested by nodes. The following equation (4) calculates the energy harvested $E_{H i, u}$ from $\mathrm{u}^{\text {th }} \mathrm{RF}$ source by the $i^{\text {th }}$ node as:

$$
E_{H i, u}=\Gamma P_{u} \frac{G_{u} G_{i} \lambda_{u}^{\alpha}}{4 \pi d_{i, u}^{\alpha}} h_{t}
$$

Here, the antenna gains achieved from $i^{\text {th }}$ node and $u^{\text {th }}$ energy harvesting source are represented as $G_{i}$ and $G_{u}$ respectively. The power the of signal that is transmitted by $u^{\text {th }}$ source of energy is denoted by $P_{u}$. The distance existing in between the $\mathrm{i}^{\text {th }}$ node and $\mathrm{u}^{\text {th }}$ energy harvesting source is denoted by $d_{i, u}$. The path-loss exponent is denoted here by $\alpha$ and the harvesting efficiency by $\Gamma$. The wavelength of RF signal is denoted by $\lambda$ and the harvesting duration by $h_{t}$.

TABLE II

SYMBOLS IN THIS PAPER

\begin{tabular}{cc}
\hline Symbols & Description \\
\hline $\mathrm{N}$ & Number of Sensor Node \\
$\mathrm{C}$ & Clusters in the network \\
$\mathrm{r}$ & Frame Index \\
$i$ & Sensor node index \\
$i_{0}$ & $\mathrm{CH}$ index
\end{tabular}

$E_{i}^{\text {cons }} \quad$ Energy consumed by node i during a single frame

$E_{i 0}^{\text {cons }} \quad$ Amount of consumed by $\mathrm{i}_{0}$ th $\mathrm{CH}$ during a single frame

$E_{H i, u} \quad$ Total harvested energy by sensor node $\mathrm{i}$ from $\mathrm{u}^{\text {th }}$ source

$\Gamma \quad$ Harvesting Efficiency

$E_{t h} \quad$ Energy Threshold

$E_{i, c} \quad$ Current/Present energy level of node i

Ei,a Average energy of node i

\section{FRAMEWORK OF ENERGY AND SPECTRUM FOR IoT}

\section{A. Energy Management Scheme (EMS)}

The EMS includes of an mode switching of the energy method and a $\mathrm{CH}$ selection method which is the most important aspect of energy management in IoT [29]-[31]. Heterogeneous sensor network comprises of sensor nodes with different capabilities, for example, different energy levels, computational 
power, sensing range, etc. In this paper, we introduced the heterogeneous network[32]-[35] in which nodes will have different energy levels and different types of nodes.

\section{1) Mode switching of the Energy:}

Energy mode technique enables nodes to choose their mode as per their present energy level. Let Ei,c indicates the current/present energy level of nodes. Fig.2 portrays the two modes for the ith node. In FCM (full capability mode), the nodes performs harvesting of energy, environmental monitoring, and other supporting task related to CRSN. The condition for FCM, the total consumed energy in a given frame should always be equal to or greater than the total harvested energy. In RCM (reduced capability mode), the nodes perform just the task of harvesting. The ith node will check its level of energy and move from FCM to RCM when energy falls down the threshold level $\mathrm{E}_{\mathrm{th}}$ as shownin figure 2.Taking RCM and FCM in consideration[21][32], two extra modes are presented known as (1) dynamic operating mode (DOM) and(2) fixed operating mode (FOM)[33]. In DOM, nodes can switch among RCM and FCM, though in FOM nodes can just work in FCM[21].

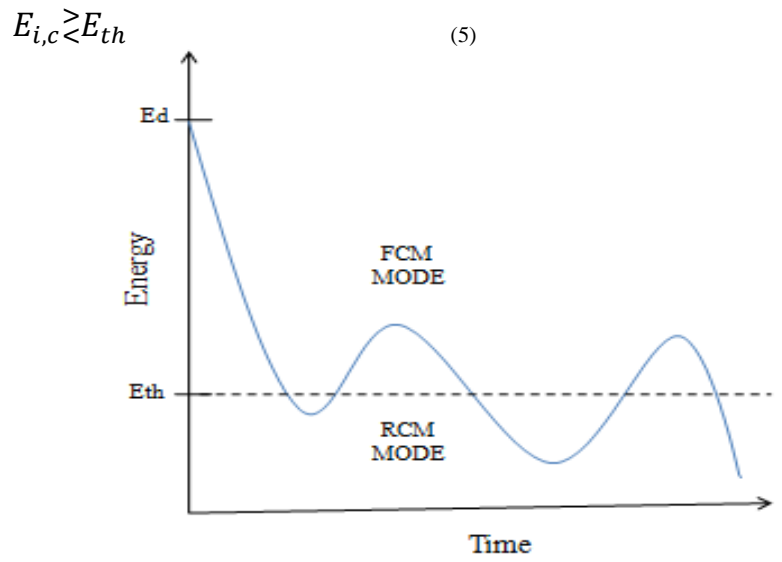

Fig.2: Different energy aware modes

\section{2) Selection of Cluster head}

The selection of $\mathrm{CH}$ accomplishes balancing of energy by choosing the energy-rich sensor node as $\mathrm{CH}$ on rotational premise for the initial frame tenure whereas for the second round another selection criteria is presented which includes past and present energy levels of nodes. Nodes may have a high level of energy at a specific time of moment by remaining longer in RCM. However, the nodes can rapidly exhaust their energy because of their high departure rate and low energy arrival rate. Consequently, by taking the past energy values into consideration, the node with a low departure rate is selected as a $\mathrm{CH}[21][36]$. The mechanism for $\mathrm{CH}$ is described below:

1) Every sensor node in the network send their EIP to the prior chosen $\mathrm{CH}$ that contains: ID of the node, average and present or current energy level during the selection frame.
2) When $\mathrm{CH}$ receives EIP from all nodes, the prior chosen $\mathrm{CH}$ maximizes Eic/Eia so as to choose the next $\mathrm{CH}$. The selection of $\mathrm{CH}$ is detailed as below:

$$
\max \frac{E_{i, c}}{E_{i, a}}
$$

Fig.3 shows the flow diagram of the reference work and the proposed work, in modified work extra gateway node, is there for long-distance communication and the cache nodes are also deployed to save the most frequent data to avoid the overconsumption of the energy. When the base station will make a request for the required data, if that data is available at cache nodes the data can be forwarded from there only otherwise the request will be forwarded to $\mathrm{CH}$.

\section{B. Channel Management Scheme (CMS)}

For selecting the best channel in terms of reliability and stability, $\mathrm{CH}$ employs a CMS. The sensor nodes are requested to transmit the monitored data once the reporting process is initiated by $\mathrm{CH}$ [20]. In response, the desired data is forwarded on the designated channel to $\mathrm{CH}$ by the nodes. The $\mathrm{CH}$ generates the sharing pattern using CMS. CR technology is included within the sensor nodes due to which the environmental monitored data is forwarded to $\mathrm{CH}$ in the reporting process through the available channels. Higher transmission power is used by PUs such that they can stay either static or mobile. A slotted structure is assumed to be followed by the primary networks [21]. A random process is followed when forwarding PUs where in each slot duration the channel state varies randomly. Every channel is characterized on the basis of PU index by $\mathrm{CH}$ on the basis of sensing reports gathered from various sensor nodes. Over various channels the $V$ recent PU activities are stored by $\mathrm{CH}$. A model with two states that includes OFF and ON state is used to model the PU behavior. Let $S$ regions each with weight $\Phi_{\mathrm{k}, \mathrm{r}}^{\mathrm{S}}$ be generated by dividing the history samples $V$. In manner that new history samples have more or higher weights in comparison to previous samples the weights are assigned. It is because of the fact that the information related to the behavior of PU is provided by the recent samples. Following equation (7) calculates the average $\mathrm{PU}$ index over $\mathrm{R}$ frames for channel $\mathrm{k}$ as:

$$
\gamma_{k}=\frac{1}{R} \sum_{r=1}^{R} \sum_{s=1}^{S} \Phi_{\mathrm{k}, \mathrm{r}}^{\mathrm{S}} \theta_{\mathrm{k}, \mathrm{r}}^{\mathrm{s}}
$$

Here, the numbers of consecutive busy states (H1) of channel k within specific history areas are indicated by $\theta_{\mathrm{k}, \mathrm{r}}^{\mathrm{s}}$. The channel is considered to be highly stable when the value of $\gamma_{k}$ is small. The PU history is incorporated by the average PU index. A channel can be detected as being idle and available in one scenario however, QoS degradation to the nodes can be caused when PU arrives on that channel. The view regarding energy of nodes and stability of channel is provided by the rationale behind the history based node and channel selection[37].Following equation (8) calculates the reliability $Q_{r, k}$ of $\mathrm{k}^{\text {th }}$ channel in frame $\mathrm{r}$ as:

$$
Q_{r, k}=\frac{1}{\beta_{i, k}}
$$




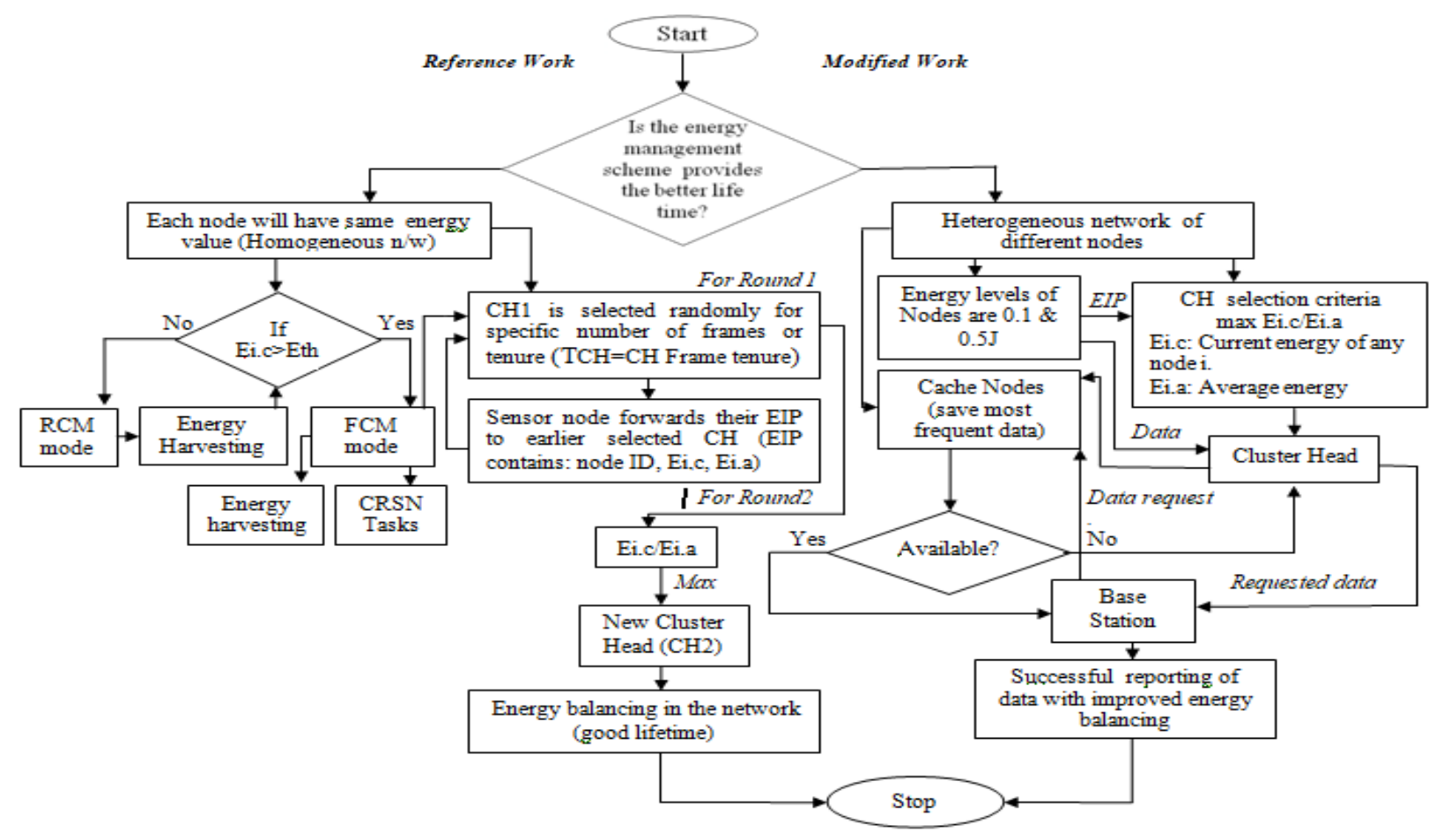

Fig.3: Flow-diagram of the proposed approach

Here, the $\mathrm{i}^{\text {th }}$ node on $\mathrm{k}$-th channel has the bit error rate (BER) denoted by $\beta_{i, k}$. The binary phase shift keying is assumed to be utilized by $\mathrm{i}^{\text {th }}$ sensor node. $\beta_{i, k}=\frac{1}{2} e^{-\xi_{b, k}}$. The energy per bit to noise power spectral density is denoted by $\xi_{b, k}$.

Maximization of the channels reliability while ensuring the PU index for all the chosen channels is the major aim of channel allocation process [21]. Based on the requirement of data transferred by nodes CMS is invoked. The formula for maximization of reliability is given as:

$$
\begin{array}{r}
\max \sum_{k=1}^{M} m_{k} Q_{r, k} \\
\text { Subjectto: } \gamma_{k} \leq W, \forall k \in\{1,2, \ldots, M\} \\
m_{k}=\{0,1\}
\end{array}
$$

Here, to check if the channel $\mathrm{k}$ is occupied by the sensor nodes or not, binary variable $m_{k}$. The maximum PU index limit up to which the channel remains stable is denoted by $\mathrm{W}$. To achieve higher stability, the channels with PU index $\left(\gamma_{k}\right)$ less than $\mathrm{W}$ are chosen as the channels are used by sensor nodes in an opportunistic manner. It means that the channels with low arrival of PU can be used for data transmission[38].

TABLE III

PARAMETERS

\begin{tabular}{ll}
\hline Parameters & Values \\
\hline
\end{tabular}

Nodes in each cluster

34

Nodes Energy

$0.1 \mathrm{~J}, 0.5 \mathrm{~J}$
Energy used in transmission $\left(\mathrm{E}_{t x}\right)$

Dissipated energy for intracluster communication $\left(\varepsilon_{\mathrm{fs}}\right)$

Dissipated energy for $\mathrm{CH}$ to sink communication $\left(\varepsilon_{\mathrm{amp}}\right)$

Size of EIP

$0.5 \mathrm{~kb}$

Modulation Scheme

DBPSK

\section{SIMULATION RESULT}

The energy efficient model is proposed in this research work. The HEACHS energy efficient protocol use the heterogeneous sensor nodes for the information sensing. The sensing information will be passed through the cache nodes to base station. In the HEACHS energy efficient protocol, cluster head send information to cache nodes which pass information to the base station. In this section, we check the functionality of new framework. The area of $700 \times 700 \mathrm{~m}$ is taken in which 500 sensors nodes are distributed uniformly. The whole area is divided into small cluster, each cluster will have equal number of nodes i.e $\mathrm{N}=20$. The performance of proposed Heterogeneous energy aware $\mathrm{CH}$ selection (HEACHS) with Energy management scheme (EMS), Current/Present \& Average energy based selection of CH (CAECHS)[19][21][[39][40], Energy aware CH selection (EACHS) is compared in Section V. In proposed HEACHS the network is heterogeneous and there are some gateway nodes as mentioned in Section IV. In EMS, $\mathrm{CH}$ is selected on the basis 
of current and average energy with energy harvesting whereas CEACHS consider present and average energy for the selection of $\mathrm{CH}$ and EACHS consider only remaining energy for the selection of $\mathrm{CH}$.

Fig.4 and Fig.5 shows the senor node index versus average residual energy for HEACHS for 250 and 3000 frames respectively and compared with EMS, CEACHS, EACHS. It is clear that the proposed HEACHS have better lifetime as compared to other schemes. Fig. 6 illustrates the frame index versus number of alive nodes for HEACHS and compared with EMS and it is clearly visible that proposed schemes have more number of alive nodes when compared with existing scheme. Fig.7 depicts the number of packet sent versus rounds. HEACHS sent more number of packets successfully when compared to the EMS.

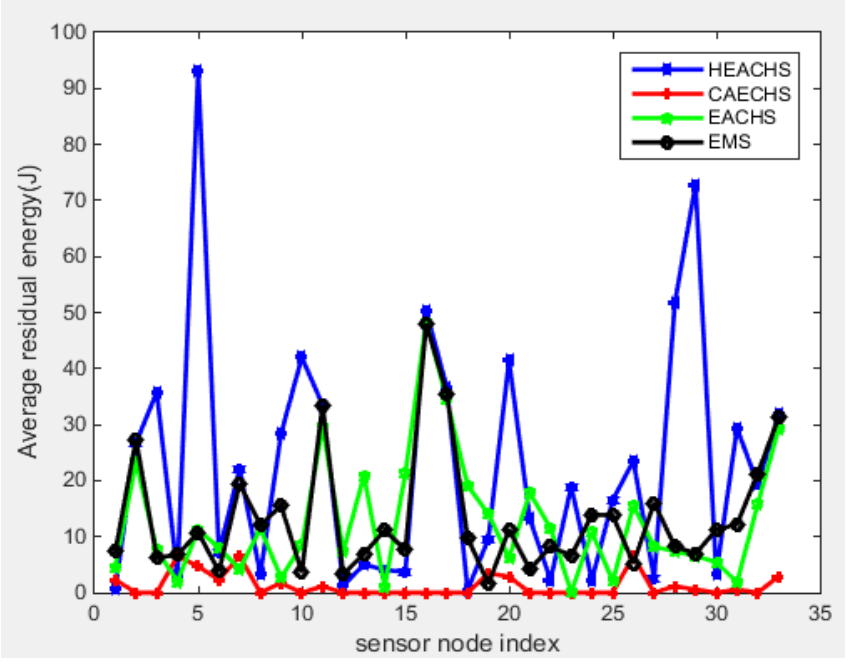

Fig.4:Average Residual Energy for 250 Frames

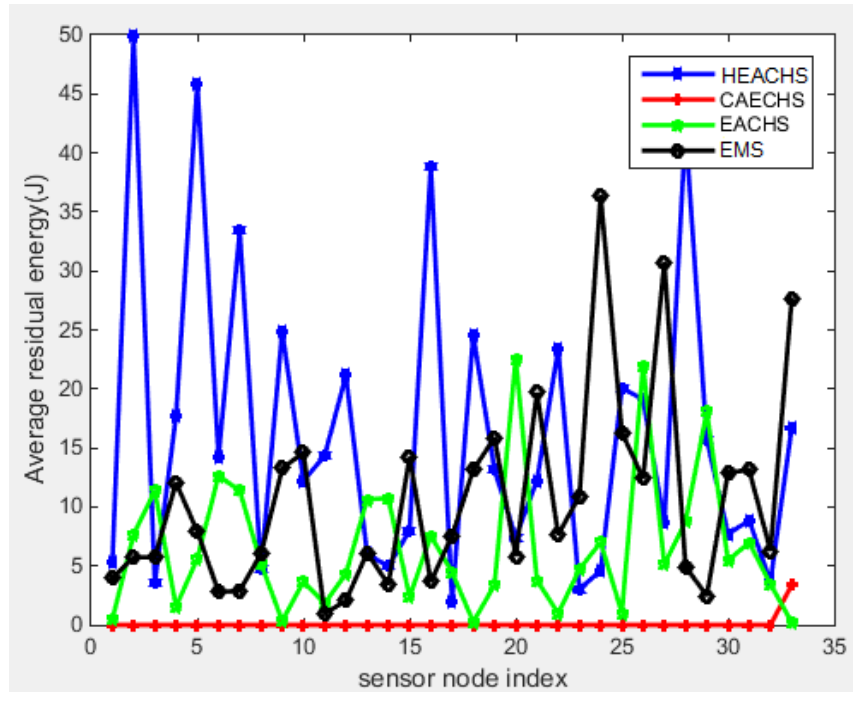

Fig.5:Average Residual Energy for 3000 Frames

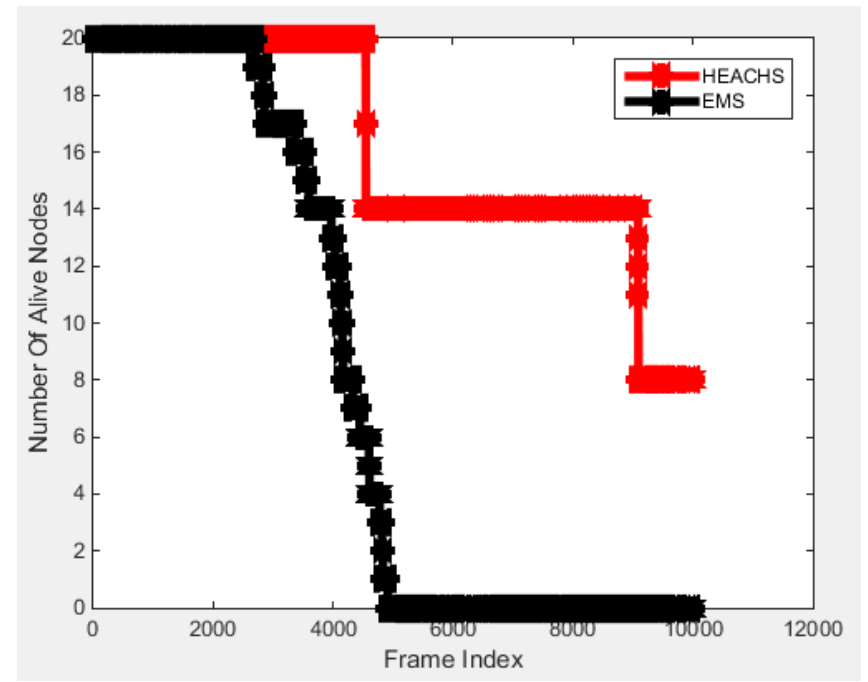

Fig.6:Alive Nodes in the Network

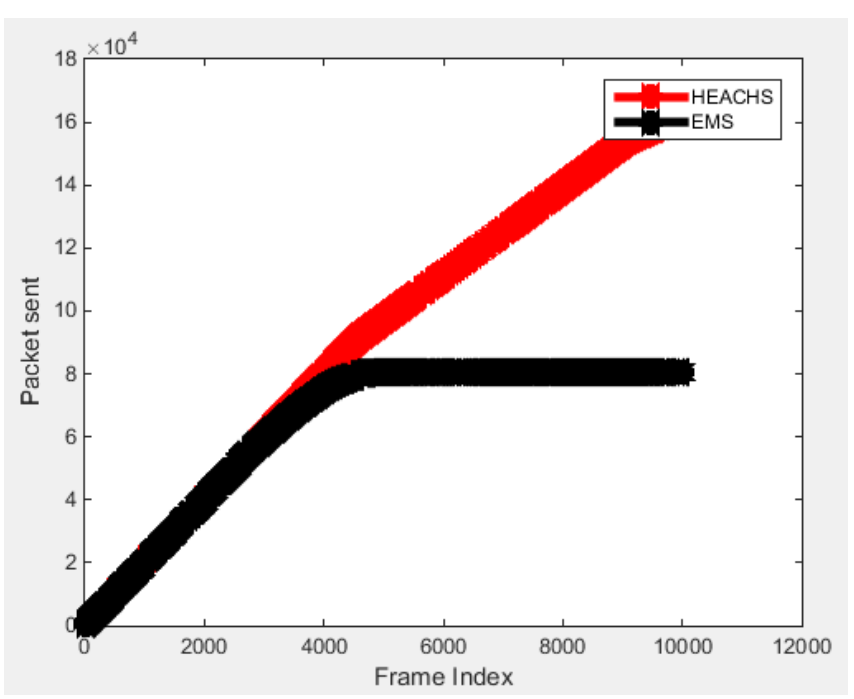

Fig.7:Number of packets sent successfully

\section{CONCLUSION}

Cognitive radio and Internet of Things have risen as promising innovations to upgrade our lives by proficiently and smartly utilizing the current resources. This paper addressed two principle difficulties to the CRSN for IoT: (1) energy efficiency and (2) spectrum/channel management. Mode switching of energy , $\mathrm{CH}$ selection algorithm, Cache nodes deployment are introduced for better energy efficiency in the network. Mode switching allows nodes to operate in two modes (FOM and DOM) accordingly. $\mathrm{CH}$ is selected on the basis of present and average of the nodes in order to balance the energy in the network. whereas, gateway nodes are there to store the most frequent data to reduce the consumption of energy in the network. Gateway nodes are deployed near to the base station which will also promote the multi hop communication. Simulation results show the expansion in the lifetime of the network in terms of functional nodes and residual energy. The proposed HEACHS shows higher alive nodes as compared to the EMS, EACHS \&CAECH schemes respectively. In, future this work can be extended to incorporate the different levels of clustering in the network.

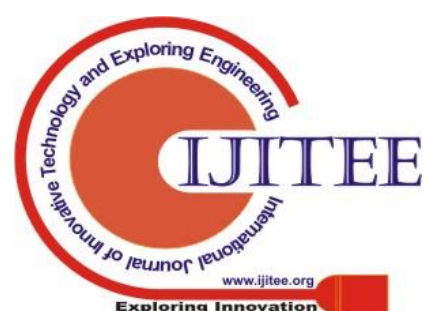




\section{Performance analysis of Cognitive Radio Based IoT Network For Energy Efficiency and Spectrum utilization}

\section{REFERENCES}

[1] A. El-Mougy, M. Ibnkahla, G. Hattab, and W. Ejaz, "Reconfigurablewireless networks," Proceedings of the IEEE, vol.103, no. 7, pp. 1125-1158, Jul. 2015.

[2] W. Ejaz and M. Ibnkahla, "Machine-to-machine communications in cognitive cellular systems," in IEEE International Conference on Ubiquitous Wireless Broadband (ICUWB). Montreal, QC: IEEE, Oct. 2015, pp. 1-5.

[3] M. Ibnkahla, Cooperative Cognitive Radio Networks: The CompleteSpectrum Cycle. CRC Press, 2014.

[4] G. Hattab and M. Ibnkahla, "Multiband spectrum access: Great promisesfor future cognitive radio networks," Proceedings of the IEEE, vol. 102,no. 3, pp. 282-306, Mar. 2014

[5] W. Ejaz, N. Ul Hasan, and H. S. Kim, "Distributed cooperative spectrumsensing in cognitive radio for ad hoc networks," Computer Communications,vol. 36, no. 12, pp. 1341-1349, Jul. 2013

[6] A. El-Mougy and M. Ibnkahla, "A cognitive framework for WSN basedon weighted cognitive maps and Q-learning," Ad Hoc Networks, vol. 16,pp. 46-69, 2014

[7]Z. Sheng, S. Yang, Y. Yu, A. V Vasilakos, J. A. Mccann, and K. K. Leung, "A Survey on The IETF Protocol Suite for The Internet-ofThings: Standards , Challenges and Opportunities," IEEE Wireless Communications, vol. 20, no. 6, pp. 91-98, 2013.

[8] J. Zhao, R. Govindan, and D. Estrin, "Residual Energy Scans for Monitoring Wireless Sensor Networks," Proceedings of IEEE Wireless Communications and Networking Conference, pp. 356-362, 2002.

[9] S. Aslam, W. Ejaz and M. Ibnkahla, "Energy and Spectral Efficient Cognitive Radio Sensor Networks for Internet of Things", IEEE Internet of Things Journal, vol. 5, no. 4, pp. 3220-3233, 2018

[10]B. Mostafa, A. Benslimane, M. Saleh, S. Kassem and M. Molnar, "An Energy-Efficient Multiobjective Scheduling Model for Monitoring in Internet of Things", IEEE Internet of Things Journal, vol. 5, no. 3, pp. 1727-1738, 2018.

[11]S. Santiago and L. Arockiam, "A novel fuzzy based energy efficient routing for Internet of Things", 2017 International Conference on Algorithms, Methodology, Models and Applications in Emerging Technologies (ICAMMAET), 2017.

[12]S. Nisha and S. Balakannan, "An energy efficient self organizing multicast routing protocol for Internet of Things", 2017 IEEE International Conference on Intelligent Techniques in Control, Optimization and Signal Processing (INCOS), 2017.

[13]K. Suresh, M. RajasekharaBabu and R. Patan, "EEIoT: Energy efficient mechanism to leverage the Internet of Things (IoT)", 2016 International Conference on Emerging Technological Trends (ICETT), 2016.

[14]M. Yasin, Q. Abuein, A. Amer and M. Qasem, "An energy-efficient technique for constrained application protocol of Internet of Things", 2016 International Conference on Engineering \& MIS (ICEMIS), 2016.

[15]W. Ejaz, M. Naeem, A. Shahid, A. Anpalagan, and M. Jo, "Efficient energy management for Internet of Things in smart cities," IEEE Communications Magazine (in press), vol. 55, no. 1, pp. 84-91, Jan 2017.

[16]R. Zhang and C. K. Ho, "MIMO broadcasting for simultaneous wireless information and power transfer," IEEE Transactions on Wireless Communications, vol. 12, no. 5, pp. 1989-2001, May 2013.

[17] Y. Liu, Y. Zhang, R. Yu, and S. Xie, "Integrated energy and spectrum harvesting for 5G wireless communications," IEEE Network, vol. 29, no. 3 , pp. 75-81, Jun. 2015.

[18]N. Panahi, H. O. Rohi, A. Payandeh, and M. S. Haghighi, "Adaptationof leach routing protocol to cognitive radio sensor networks" in SixthInternational Symposium on Telecommunications (IST). Tehran, Iran:IEEE, Nov. 2012, pp. 541-547.

[19]J.-S. Leu, T.-H. Chiang, M.-C. Yu, and K.-W. Su, "Energy efficientclustering scheme for prolonging the lifetime of wireless sensor networkwith isolated nodes," IEEE communications letters, vol. 19, no. 2, pp.259-262, Feb. 2015.

[20] N. Jain and V. A. Bohara, "Energy harvesting and spectrum sharingprotocol for wireless sensor networks," IEEE Wireless CommunicationsLetters, vol. 4, no. 6, pp. 697-700, Dec. 2015.

[21]S. Aslam, W. Ejaz and M. Ibnkahla, "Energy and Spectral Efficient Cognitive Radio Sensor Networks for Internet of Things", IEEE Internet of Things Journal, vol. 5, no. 4, pp. 3220-3233, 2018. Available: 10.1109/jiot.2018.2837354.

[22] S. K. Devi, "Survey On Sleep Scheduling Methods In Wireless Sensor Networks," International Journal of Advanced Research in Computer Engineering \& Technology (IJARCET), vol. 3, no. 1, pp. 81-85, 2014.

[23] P. Sasikumar and S. Khara, "K-means clustering in wireless sensor networks," in Fourth international conference on Computational intelligence and communication networks (CICN). Mathura, India: IEEE, Nov. 2012, pp. 140-144

[24] Y. Chen and Q. Zhao, "On the lifetime of wireless sensor networks, IEEE
Communications letters, vol. 9, no. 11, pp. 976-978, Nov. 2005

[25] Z. Cheng, M. Perillo, and W. B. Heinzelman, "General network lifetime and cost models for evaluating sensor network deployment strategies,"IEEE Transactions on mobile computing, vol. 7, no. 4, pp. 484-497, Apr. 2008

[26] A. Woo, T. Tong, and D. Culler, "Taming the underlying challenges of reliable multihop routing in sensor networks," in Proceedings of the first international conference on Embedded networked sensor systems SenSys '03, 2003, pp. 14-27.

[27] S. Raza, L. Wallgren, and T. Voigt, "SVELTE: Real-time intrusion detection in the Internet of Things," Ad Hoc Networks, vol. 11, no. 8, pp. 2661-2674, 2013.

[28] R. Giuliano, F. Mazzenga, A. Neri, and A.M. Vegni, "Security Access Protocols in IoT Capillary Networks," in IEEE Internet of Things Journal, Vol. 4, no. 3, pp. 645-657, June 2017.

[29]J. Stankovic, "Research Directions for the Internet of Things", IEEE Internet of Things Journal, vol. 1, no. 1, pp. 3-9, 2014.

[30]X. Lu, P. Wang, D. Niyato, D. I. Kim, and Z. Han, "Wireless networks with RF energy harvesting: A contemporary survey," IEEE Communications Surveys \& Tutorials, vol. 17, no. 2, pp. 757-789, May 2015

[31] H. Kharrufa, H. Al-Kashoash, Y. Al-Nidawi, M. Q. Mosquera, and A. H. Kemp, "Dynamic RPL for multi-hop routing in IoT applications," in Proceedings of the 13th Annual Conference on Wireless On-Demand Network Systems and Services (WONS '17), pp. 100-103, February 2017.

[32] A. Aijaz and A. H. Aghvami, "Cognitive machine-to-machine communications for internet-of-things: a protocol stack perspective," IEEE Internet of Tings Journal, vol. 2, no. 2, pp. 103-112, 2015.

[33] S. Basagni, C. Petrioli, R. Petroccia, and D. Spaccini, "CARP: a channelaware routing protocol for underwateracoustic wireless networks," Ad Hoc Networks, vol. 34, no. supplement C,pp. 92-104,2015, Advances in Underwater Communications and Networks.

[34] C. Liu, C. Yang, X. Zhang, and J. Chen, "External integrity verifcation for outsourced big data in cloud and IoT: a big picture," Future Generation Computer Systems, vol. 49, no. supplement C, pp. 58-67, 2015.

[35]W. Ejaz, M. Naeem, M. Basharat, S. Kandeepan, and A. Anpalagan, "Efficient wireless power transfer in software-defined wireless sensor networks," IEEE Sensors Journal, vol. 16, no. 20, pp. 7409-7420, Oct 2016.

[37] X. Lu, P. Wang, D. Niyato, D. I. Kim, and Z. Han, "Wireless networks with RF energy harvesting: A contemporary survey," IEEE Communications Surveys \& Tutorials, vol. 17, no. 2, pp. 757-789, May 2015.

[37]W. Ejaz, G. A. Shah, H. S. Kim et al., "Energy and throughput efficient cooperative spectrum sensing in cognitive radio sensor networks, "Transactions on Emerging Telecommunications Technologies, vol. 26, no. 7, pp. 1019-1030, Jul. 2015.

[38] T. H. Cormen, Introduction to algorithms. MIT press, 2009.

[39] M. Mishra, C. R. Panigrahi, B. Pati, and J. L. Sarkar, "ECHS: An energy aware cluster head selection algorithm in Wireless Sensor Networks," in International Conference on Man and Machine Interfacing (MAMI). Bhubaneswar, India: IEEE, Dec. 2015, pp. 1-4.

[40] V. Pal, G. Singh, and R. Yadav, "Energy efficient cluster head selection scheme: A change in node death scenario of leach for surveillance wireless sensor networks," in IEEE International Conference on Parallel Distributed and Grid Computing (PDGC). Himachal Pradesh, India ,IEEE, Dec. 2012, pp. 865-869.

\section{AUTHORS PROFILE}

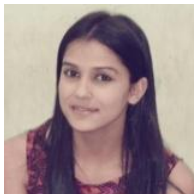

Palak Thakur received her B.Tech degree in Electronics and Communication Engineering from Baddi University of Emerging Sciences and Technology, Himachal Pradesh, India in 2017. She is currently a M.E student at Chandigarh University, Punjab, India in the department of Electronics and Communication since 2017. Her area interest of are Wireless sensor networks, Cognitive Radio and Internet-of-Things. 


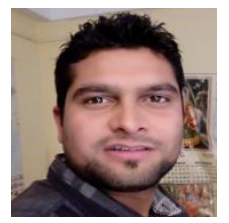

Dr. Prabhat Thakuris a Post Doctoral Research Fellow at University of Johannesburg, South Africa. He received his B.Tech degreefrom Singhania University, Rajasthan and. He completed his $\mathrm{M} \mathrm{PhD}$ in the Department of Electronics and Communication Engineering from Jaypee University of Information and Technology, Himachal Pradesh. He has many Publications in different areas of research. He also worked as a Assistant Professor at Chandigarh University in Electronics and Communication Engineering. His area of interest are Cognitive Radio, Internet- of-Things, Wireless Sensor Networks.

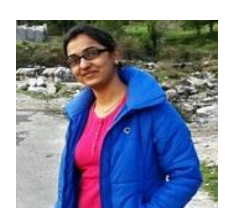

Payal Patial was born in Palampur, Himachal Pradesh, India, in 1989. She received her B.Tech and M.Tech in Electronics and Communication Engineering from Lovely Professional University, Punjab, India in the year 2011 and 2013 respectively. She is working at Chandigarh University as Assistant Professor in Electronics and Communication Engineering since 2013. Her research interest includes Biomedical Signal Processing 